\title{
THE DEGREE AND CLASS OF MULTIPLY TRANSITIVE GROUPS*
}

\author{
BY \\ W. A. MANNING
}

If a group contains substitutions of degree $\mu$ but none that displace fewer letters, the identity excepted, $\mu$ is said to be its class. $\dagger$ The same term is unfortunately used with a different meaning in the theory of prime power groups.

In substitution group theory the two problems of the order and of the class of groups of given degree are in large measure interchangeable. $\neq$ For this reason Bochert's inequalities, limiting the degree $n$ of a multiply transitive group in terms of its class $\mu$ are second in importance only to Sylow's theorem. $\S$ Bochert's theorem may be stated as follows. I

The class ( $>3$ ) of a substitution group of degree $n$ exceeds $\frac{1}{3} n-\frac{2}{3} \sqrt{n}$ if it is doubly, $\frac{1}{3} n-1$ if triply, $\frac{1}{2} n-2$ if quadruply, transitive.

In the proof of this theorem Bochert introduces the condition that the class is a given number by means of the commutator of two non-commutative substitutions. Now the commutator is only one combination out of many that may be made from the substitutions of a group $(G)$ and is by no means always one of the substitutions of lowest degree in $G$. In the hope of penetrating more deeply into this matter the author has assumed the presence of substitutions of order 2 among the substitutions of lowest degree in $G$ and thereby gains the advantages that are afforded by the simplicity of structure of diedral rotation groups. The principal result arrived at may be put thus:

1. Theorem. Let $n$ be the degree and $\mu(>2)$ the class of a group that contains a substitution of order 2 and degree $\mu$; then if it is

$$
\begin{aligned}
& \text { doubly transitive, } \quad \mu>\frac{1}{2} n-\frac{1}{2} \sqrt{n}-1 \text {, } \\
& \text { triply " , , } \mu \geqq \frac{1}{2} n \text {, } \\
& \text { 4-ply “ , } \quad \mu \geqq \frac{1}{2}(n+1) \text {, } \\
& \text { 5-ply } \quad \text { " } \quad, \quad \mu \geqq \frac{3}{5} n \text {, } \\
& \text { 6-ply “ } \quad, \quad \mu>\frac{3}{4} n \text {, } \\
& 7-p l y \quad \text { " , } \quad \mu \geqq \frac{1}{1} \frac{0}{3} n,
\end{aligned}
$$

* Presented to the Society, Nov. 25, 1916.

† Jordan, Paris C o m p te s R e nd us, vol. 72 (1871), p. 384.

$\ddagger$ See these Transactions, vol. 16 (1915), p. 139.

§ Jordan, J o u rnal de mathématiques, ser. 5, vol. 1 (1895), p. 35.

\| Bochert, M athematis che Annalen, vol. 40 (1892), p. 176; vol. 49 (1897), p. 133.

Trans. Am. Math. Soc. 30 
more than $p_{1}+p_{2}+\cdots+p_{r}$ times transitive, where $p_{1}, p_{2}, \cdots, p_{r}$ are distinct odd primes $(r>1)$,

$$
\mu>\frac{\left(p_{1}-1\right)\left(p_{2}-1\right) \cdots\left(p_{r}-1\right)-1}{\left(p_{1}-1\right)\left(p_{2}-1\right) \cdots\left(p_{r}-1\right)} n .
$$

\section{The DIEDRAL GROUPS}

2. The auxiliary theorem by means of which this will be proved is the following:

If the order of a group of class $\mu$ generated by two substitutions $s$ and $t$ of order 2 and degree $\mu$ is divisible by each of the odd primes $p_{1}, p_{2}, \cdots, p_{r}$, its degree $n$ does not exceed $\mu+\mu /\left(p_{1}-1\right)\left(p_{2}-1\right) \cdots\left(p_{r}-1\right)$; if the order $N$ of st is odd or is divisible by $4, n$ does not exceed $\mu+2 \mu / N$; if $N(>2)$ is twice an odd number, $n$ is not greater than $\mu+2 \mu / N+8 \mu / N^{2}$.

If we erase from $\{s, t\}$, of degree $n$, the $\xi$ letters of all the regular constituents of $\{s, t\}$ which involve letters displaced by both $s$ and $t$ the group in the remaining constituents is of degree $n-\xi$, of class $\mu-\xi$, and is generated by two similar substitutions of order 2 and degree $\mu-\xi$. Then if the theorem can be shown to be true for diedral groups satisfying the given conditions, but in which the regular constituents, if any, are of order 2 and exhibited by transpositions of $s$ (or $t$ ) in letters fixed by $t$ (or $s$ ), it is $a$ fortiori true when regular constituents on letters common to $s$ and $t$ are present. It may be noted that if $p^{a}$ is a Sylow divisor of $N$, i. e., the highest power of the prime $p$ that divides the order of $s t$, and if $p^{a}$ divides the degree of a regular constituent of $\{s, t\}$ it must also divide the degree of some non-regular constituent of $\{s, t\}$. If this is not so, $(s t)^{N / p}$ involves no letter not in a regular constituent of $\{s, t\}$ and is not the identity. Thus the degree of $(s t)^{N / p}$ is at least $\mu$ and cannot exceed $\xi$; but if $\xi=\mu$ the truth of the theorem is not in dispute. Consequently the deletion of the regular constituents from the group $\{s, t\}$ leaves a group of the same order. In what follows the group $\{s, t\}$ under discussion will be assumed free from any regular constituent on letters common to $s$ and $t$.

The transitive constituents of $\{s, t\}$ may be enumerated as follows. In $s$ there are $m_{1}$ cycles that displace letters not in $t$, and $t$ has $m_{2}$ cycles that displace letters not in $s$; let $m=m_{1}+m_{2}$. There are $y_{j}$ constituents of degree $Y_{j}$ and order $2 Y_{j}, Y_{j}$ an odd number, $j=1,2, \cdots$. There are $z_{k}^{\prime}$ transitive constituents of degree $Z_{k}$ and order $2 Z_{k}, Z_{k}$ even, $k=1,2, \cdots$, with the generator of degree $Z_{k}$ in $s$ and the generator of degree $Z_{k}-2$ in $t$. In like manner there are $z_{k}^{\prime \prime}$ constituents of the same order $Z_{k}$ with $Z_{k}-2$ letters in $s$ and $Z_{k}$ letters in $t$; let $z_{k}=z_{k}^{\prime}+z_{k}^{\prime \prime}$. The numbers $Y_{1}, Y_{2}, \cdots$ are all the odd divisors of $N$, unity excepted, and the numbers $Z_{1}, Z_{2}, \cdots$ 
are all the even divisors of $N$, with the exception of 2 . We recall that a nonregular transitive diedral rotation group of degree $n$ is of class $n-1$ if $n$ is odd, and is of class $n-2$ if $n$ is even. In the latter case the two generators of order 2 are not similar substitutions. Of course some of the numbers $y_{1}, y_{2}, \cdots, z_{1}^{\prime}, z_{2}^{\prime}, \cdots, z_{1}^{\prime \prime}, z_{2}^{\prime \prime}, \cdots$ may be zero. The degree of $s$ is

$$
2 m_{1}+\sum \cdot y_{j}\left(Y_{j}-1\right)+\sum z_{k}^{\prime} Z_{k}+\sum z_{k}^{\prime \prime}\left(Z_{k}-2\right),
$$

and the degree of $t$ is

$$
2 m_{2}+\sum y_{j}\left(Y_{j}-1\right)+\sum z_{k}^{\prime}\left(Z_{k}-2\right)+\sum z_{k}^{\prime \prime} Z_{k} .
$$

The summations extend the subscripts through their entire range of values. With this understanding we may omit the subscripts $j$ and $k$ in future and introduce these abbreviations:

and similarly

$$
E_{1} \equiv \sum y(Y-1), \quad F_{1} \equiv \sum z(Z-1),
$$

$$
\Gamma_{a} \equiv E_{a}+F_{a} \equiv \sum y(Y-a)+\sum z(Z-a) .
$$

Then the degree of $\{s, t\}$ is $2 m+\Gamma_{0}$ which on the elimination of $2 m$ becomes $2 \mu-\Gamma_{2}$; for

$$
\begin{aligned}
-2 \Gamma_{1}+\Gamma_{0}=\sum y Y-\sum(2 y Y-2 y)+\sum z Z-\sum(2 z Z-2 z) & \\
= & -\sum y(Y-2)-\sum z(Z-2)=-\Gamma_{2} .
\end{aligned}
$$

3. If $s t$ is of odd prime order it is obvious that $n=\mu+\mu /(p-1)$.

Let $s t$ be of order $p^{\alpha}, p$ any prime and $\alpha>1$. Let $\{s, t\}$ have $x_{i}$ transitive constituents of degree $p^{i}, i=1,2, \cdots, \alpha$. From $(s t)^{p^{\alpha-1}}$ we get the equation,

from $(s t)^{p^{a-2}}$,

$$
p^{a} x_{a}=\mu+h_{a},
$$

from $(s t)^{p^{\alpha-3}}$,

$$
p^{a-1} x_{a-1}+p^{a} x_{a}=\mu+h_{a-1},
$$

$$
p^{a-2} x_{a-2}+p^{a-1} x_{a-1}+p^{a} x_{a}=\mu+h_{a-2},
$$

from $(s t)^{p}$,

while

$$
p^{2} x_{2}+p^{3} x_{3}+\cdots+p^{a} x_{a}=\mu+h_{2},
$$

and

$$
p x_{1}+p^{2} x_{2}+\cdots+p^{a} x_{a}=\mu+\delta,
$$

$$
(p-1) x_{1}+\left(p^{2}-1\right) x_{2}+\cdots+\left(p^{a}-1\right) x_{a}=\mu \text {. }
$$

Here $h_{2}, h_{3}, \cdots, h_{a}, \delta$, are positive integers or zero, and $\mu+\delta$ is the degree 
of $\{s, t\}$. These $\alpha+1$ equations are consistent, their eliminant must vanish, and when equated to zero and reduced it gives

$$
\delta=\mu / p^{\alpha-1}(p-1)-\sum_{i=2}^{a} h_{i} / p^{i-1} .
$$

Then if st is of order $p^{a}(\alpha>1)$, the degree of $\{s, t\}$ does not exceed

$$
\mu+\mu / p^{\alpha-1}(p-1) \text {. }
$$

4. Let $N=2 p^{a}$, where $p^{a}$ is a power of an odd prime. Let $y$ and $z$ be the number of transitive constituents of degrees $p^{a}$ and $2 p^{a}$ respectively in $\{s, t\}$. Then from $(s t)^{N / p}$ and $(s t)^{N / 2}$ we have

and

$$
\begin{aligned}
p^{a} y+2 p^{a} z & =\mu+h, \\
2 m \quad+2 p^{a} z & =\mu+k-\bar{F}_{0},
\end{aligned}
$$

with

$$
2 m+p^{a} y+2 p^{a} z=\mu+\delta-\bar{\Gamma}_{0},
$$

$$
m+\left(p^{\alpha}-1\right) y+\left(2 p^{\alpha}-1\right) z=\mu-\bar{\Gamma}_{1} .
$$

By $\bar{F}_{0}, \bar{\Gamma}_{0}$, and $\Gamma_{1}$ is meant the summation explained above but with the exclusion of constituents whose degree is divisible by $p^{a}$. The meaning of $h, k$, and $\delta$ is the same as before. They are positive integers or zero. The eliminant of $m, y$, and $z$ is

This reduces to

$$
\left|\begin{array}{cccc}
0 & p^{a} & 2 p^{a} & \mu+h \\
2 & 0 & 2 p^{a} & \mu+k-\bar{F}_{0} \\
2 & p^{a} & 2 p^{a} & \mu+\delta-\bar{\Gamma}_{0} \\
1 & p^{a}-1 & 2 p^{a}-1 & \mu-\bar{\Gamma}_{1}
\end{array}\right|=0 .
$$

$$
\left(p^{a}-1\right) \delta=\mu-h\left(p^{a}-1\right)-k-\left(\bar{E}_{0}+p^{a} \bar{\Gamma}_{2}\right),
$$

whence we conclude that when the order of st is twice a power of an odd prime, its degree does not exceed $\mu+\mu /\left(p^{a}-1\right)$.

5. Let $N=p_{1} p_{2} \cdots p_{r}$, the product of $r$ distinct odd prime factors.

There is at once the equation

$$
E_{1}=\mu,
$$

and there are $\sum_{i=1}^{i=r} C_{i}=2^{r}-1$ equations given by the $2^{r}-1$ powers $(s t)^{N /\left(p_{i} p_{j} \ldots\right)}$, which is in no case the identity.but is of degree $\mu+h_{i j} \ldots$, where the quantities $h_{i j} \ldots$ are positive whole numbers or zero. Let $\delta \equiv h_{12 \ldots r}$, a constantly recurring quantity since $\mu+\delta$ is the degree of $s t$ or of $\{s, t\}$. Now $(s t)^{N / p_{i} p_{j}}$, say, involves letters from and only from those transitive con- 
stituents of $\{s, t\}$ whose degree contains $p_{i}$ or $p_{j}$ as a factor, and so on for other combinations of the primes $p_{1}, p_{2}, \cdots$. The equation given by $(s t)^{N / p_{1} p_{2}}$ when $r=3$, for example, is

$$
p_{1} y_{1}+p_{2} y_{2}+p_{1} p_{2} y_{12}+p_{1} p_{3} y_{13}+p_{2} p_{3} y_{23}+p_{1} p_{2} p_{3} y_{123}=\mu+h_{12} \text {. }
$$

There are $2^{r}-1$ variables $y_{1}, \cdots, y_{12}, \cdots$ in the $2^{r}$ equations. Replace these equations by an equivalent system got by replacing the first, $E_{1}=\mu$, by $E_{0}-E_{1}=\delta$ and the last $2^{r}-2$ by themselves less the second, $E_{0}=\mu+\delta$. The eliminant of these equations is

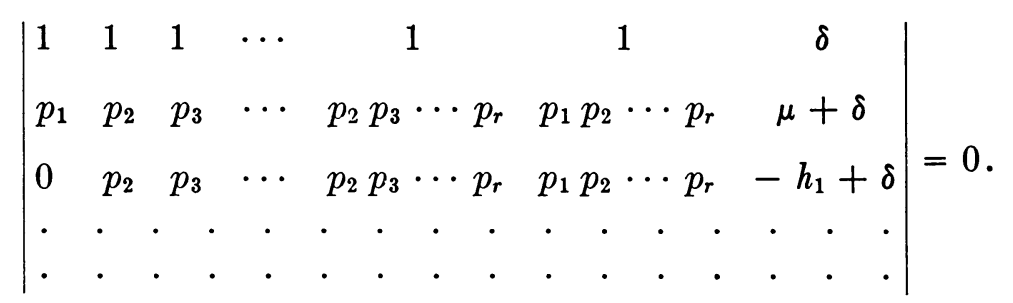

Multiply the first row of this determinant by $p_{1} p_{2} \cdots p_{r}$ and then divide the first column by $p_{1}$, the second by $p_{2}, \cdots$, the $(r+1)$ th by $p_{1} p_{2}, \cdots$. Then we have

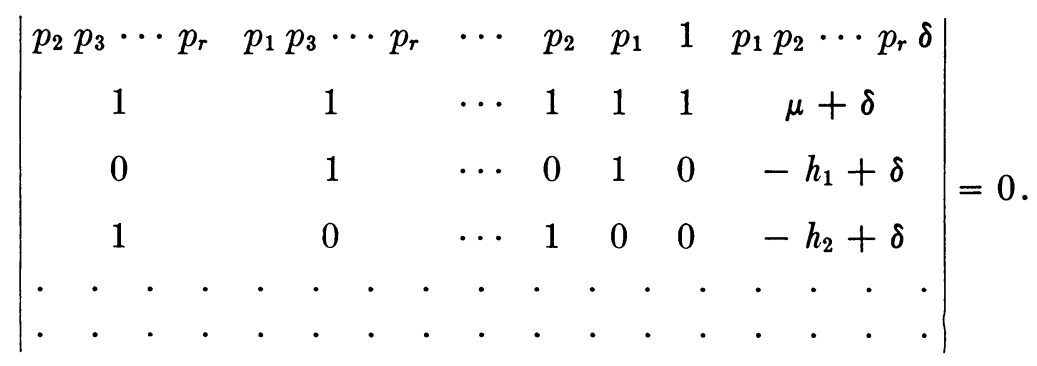

For the sake of convenience of reference we may call the row in which $h_{i}$, $h_{i j}, \cdots$ occur the $h_{i}$-row, the $h_{i j}$-row, and so forth. Then the law of the formation of the last $2^{r}-2$ rows is evident: a term in the $h_{i}$-row (except $\delta-h_{i}$ ) is unity if the term above it in the first row contains $p_{i}$ as a factor; a term in the $h_{i j}$-row is unity if the term in the first row above it contains $p_{i} p_{j}$ as a factor, and so on; otherwise it is zero. For wherever a zero is found in the $h_{i j k}$-row of the determinant there was a term $p_{i} p_{j} p_{k} y_{i j k}$ in the original equation, from which was subtracted $E_{0}=\mu+\delta$, and this product $p_{i} p_{j} p_{k}$ we divided out from every term of the column corresponding to $y_{i j k}$, thus leaving $p_{1} p_{2} \cdots p_{r} /\left(p_{i} p_{j} p_{k}\right)$ in the first row directly above the zero in question. The present units correspond to zeros in the original equations. For the sake of clearness it may be well to write out the determinant for the case $r=3$ : 


$$
\left|\begin{array}{cccccccc}
p_{3} p_{2} & p_{3} p_{1} & p_{2} p_{1} & p_{3} & p_{2} & p_{1} & 1 & p_{1} p_{2} p_{3} \delta \\
1 & 1 & 1 & 1 & 1 & 1 & 1 & \mu+\delta \\
0 & 1 & 1 & 0 & 0 & 1 & 0 & \delta-h_{1} \\
1 & 0 & 1 & 0 & 1 & 0 & 0 & \delta-h_{2} \\
1 & 1 & 0 & 1 & 0 & 0 & 0 & \delta-h_{3} \\
0 & 0 & 1 & 0 & 0 & 0 & 0 & \delta-h_{12} \\
0 & 1 & 0 & 0 & 0 & 0 & 0 & \delta-h_{13} \\
1 & 0 & 0 & 0 & 0 & 0 & 0 & \delta-h_{23}
\end{array}\right|=0 .
$$

Now multiply the second row by -1 , the $h_{i}$-row by $-\left(p_{i}-1\right)$, the $h_{i j}$-row by $-\left(p_{i}-1\right)\left(p_{j}-1\right), \cdots$, and add them all to the first row. The sum in a typical column, the $y_{12 \ldots k}$-column, is

$$
\begin{aligned}
p_{1} p_{2} \cdots & p_{k}-\left[1+\sum_{i=1}^{k}\left(p_{i}-1\right)+\sum_{i=1 ; j=i+1}^{i=k ; j=k}\left(p_{i}-1\right)\left(p_{j}-1\right)+\cdots\right. \\
& \left.+\left(p_{1}-1\right)\left(p_{2}-1\right) \cdots\left(p_{k}-1\right)\right] \\
& =p_{1} p_{2} \cdots p_{k}-\left(p_{1}-1+1\right)\left(p_{2}-1+1\right) \cdots\left(p_{k}-1+1\right)=0 .
\end{aligned}
$$

This gives zero in every term of the first row except one. From the addition of the elements of the last column we have

$$
\begin{aligned}
\delta\left[p_{1} p_{2} \cdots p_{r}\right. & -1-\sum\left(p_{1}-1\right)-\sum\left(p_{1}-1\right)\left(p_{2}-1\right)-\cdots \\
& \left.-\sum\left(p_{1}-1\right)\left(p_{2}-1\right) \cdots\left(p_{r-1}-1\right)\right] \\
& -\mu+\sum h_{1}\left(p_{1}-1\right)+\sum h_{12}\left(p_{1}-1\right)\left(p_{2}-1\right)+\cdots=0 .
\end{aligned}
$$

Hence when the order of st is the product of $r$ distinct odd primes $p_{1}, p_{2}, \cdots, p_{r}$, the degree of $\{s, t\}$ does not exceed

$$
\mu+\mu /\left(p_{1}-1\right)\left(p_{2}-1\right) \cdots\left(p_{r}-1\right) .
$$

6. Let $N=2 p q$, where $p$ and $q$ are two distinct odd primes. The following five equations can be written down:

$$
\begin{array}{lc}
\text { from } s \text { and } t \text { jointly, } & m+\Gamma_{1}=\mu, \\
\text { from } s t, & 2 m+\Gamma_{0}=\mu+\delta, \\
\text { from }(s t)^{p q}, & 2 m+F_{0}=\mu+h, \\
\text { from }(s t)^{2 q}, & \Gamma_{0}-q y_{2}-2 q z_{2}=\mu+i, \\
\text { from }(s t)^{2 p,} & \Gamma_{0}-p y_{1}-2 p z_{1}=\mu+j,
\end{array}
$$


where $\delta, h, i$, and $j$ are positive whole numbers or zero. The elimination of $m, y_{1}, z_{1}$, and $z_{3}$ gives

$$
\left|\begin{array}{ccccc}
1 & p-1 & 2 p-1 & 2 p q-1 & \mu-(q-1) y_{2}-(p q-1) y_{3}-(2 q-1) z_{2} \\
2 & p & 2 p & 2 p q & \mu+\delta-q y_{2}-p q y,-2 q z_{2} \\
2 & 0 & 2 p & 2 p q & \mu+h-2 q z_{2} \\
0 & p & 2 p & 2 p q & \mu+i-p q y_{3} \\
0 & 0 & 0 & 2 p q & \mu+j-q y_{2}-p q y_{3}-2 q z_{2}
\end{array}\right|=0
$$

which reduces to

$-\mu+q(p-1) \delta+q h+(p-1) q i+(q-1) j+q(p q-2 p+1) y_{2}$

$$
+p q(q-1) y_{3}+2 q(q-1)(p-1) z_{2}=0 ;
$$

whence $\delta \leqq \mu / q(p-1)$. If the order of st is twice the product of two distinct odd primes the degree of $\{s, t\}$ does not exceed

$$
\mu+\mu /[q(p-1)] .
$$

This case is needed in the proof of our theorem only when one of the two odd primes is 3 .

7. Now let $N$ have at least two prime power factors the smaller of which is greater than 3. $N=p^{\alpha} q^{\beta} \phi$, where $p$ and $q$ are distinct primes and $\phi$ is unity or a number relatively prime to $p$ and $q$. We start from

$$
\mu+\delta=2 \mu-\Gamma_{2} .
$$

The powers $(s t)^{N / p}$ and $(s t)^{N / q}$ give

$$
\begin{aligned}
& \Gamma_{0}(*, 0)+\Gamma_{0}(*, *)=\mu+h, \\
& \Gamma_{0}(0, *)+\Gamma_{0}(*, *)=\mu+i,
\end{aligned}
$$

where $h$ and $i$ are positive whole numbers or zero, and $\Gamma_{a}(*, 0)$ is to be understood as equal to $\sum y(Y-a)+\sum z(Z-a)$, the summation extending however only to those constituents of $\{s, t\}$ whose degrees are divisible by $p^{a}$ and not by $q^{\beta}$. In $\Gamma_{a}(0, *)$ the summation extends to the constituents whose degrees are divisible by $q^{\beta}$ and not by $p^{\alpha}$, while in $\Gamma_{a}(*, *)$ the summation extends to constituents whose degrees are divisible by both $p^{a}$ and $q^{\beta} \quad$ Thus

Then

$$
\Gamma_{a}=\Gamma_{a}(*, *)+\Gamma_{a}(*, 0)+\Gamma_{a}(0, *)+\Gamma_{a}(0,0) \text {. }
$$

$$
0=-\mu-\frac{h+i}{2}+\frac{\Gamma_{0}(*, 0)+\Gamma_{0}(0, *)}{2}+\Gamma_{0}(*, *) \text {. }
$$


Multiply by $\left(p^{\alpha} q^{\beta}-2\right) /\left(p^{\alpha} q^{\beta}\right)$ and add to

$$
\mu+\delta=2 \mu-\Gamma_{2}(*, 0)-\Gamma_{2}(0, *)-\Gamma_{2}(*, *)-\Gamma_{2}(0,0),
$$

with the result (writing $\pi$ for $p^{a} q^{\beta}$ )

$$
\mu+\delta=\mu+2 \mu / \pi-\frac{\pi-2}{\pi} \frac{h+i}{2}-\frac{\pi+2}{2 \pi} \Gamma_{4 \pi /(\pi+2)}^{\prime}-\frac{2}{\pi} \Gamma_{\pi}^{\prime \prime}-\Gamma_{2}^{(0)} .
$$

Here the new symbol $\Gamma_{a}^{(k)}$ is introduced $(k=0,1,2, \ldots)$. It is to mean the $\operatorname{sum} \Gamma_{a}(*, 0)+\Gamma_{a}(0, *)$ when $k=1, \Gamma_{a}(*, *)$ when $k=2$, $\Gamma_{a}(0,0)$ when $k=0$. It will presently be used when more than two prime powers are in question. Then $\Gamma_{a}^{(3)}$, for example, shall be the sum of all the quantities which, like $\Gamma_{a}(*, *, *, 0, \cdots)$ contain three stars in the parenthesis. Returning to the proof of the last result,

$$
\begin{aligned}
\frac{\pi-2}{2 \pi} \Gamma_{0}(*, 0)- & \Gamma_{2}(*, 0)=\sum\left(\frac{\pi-2}{2 \pi} Y-Y+2\right) y \\
& +\sum\left(\frac{\pi-2}{2 \pi} Z-Z+2\right) z=-\sum\left(\frac{\pi+2}{2 \pi} Y-2\right) y \\
& -\sum\left(\frac{\pi+2}{2 \pi} Z-2\right) z=-\frac{\pi+2}{2 \pi} \sum\left(Y-\frac{4 \pi}{\pi+2}\right) y \\
& -\frac{\pi+2}{2 \pi} \sum\left(Z-\frac{4 \pi}{\pi+2}\right) z=-\frac{\pi+2}{2 \pi} \Gamma_{4 \pi /(\pi+2)}(*, 0) ;
\end{aligned}
$$

and similarly

$$
\frac{\pi-2}{\pi} \Gamma_{0}(*, *)-\Gamma_{2}(*, *)=-\frac{2}{\pi} \Gamma_{\pi}(*, *)=-\frac{2}{\pi} \Gamma_{\pi}^{\prime \prime} .
$$

The summations $\sum$ are to be taken in accordance with the symbol $\Gamma_{0}(*, 0)$ or $\Gamma_{0}(*, *)$. Hence in this case $n \leqq \mu+2 \mu / \pi$.

8. If $q^{\beta}=3$ and $p^{\alpha}>3$, we may proceed as follows:

$$
\begin{aligned}
& n=2 \mu-\Gamma_{2}+\frac{4}{3 p^{a}}\left(-\mu-h+\Gamma_{0}(*, 0)+\Gamma_{0}(*, *)\right) \\
& +\frac{p^{a}-2}{p^{a}}\left(-\mu-i+\Gamma_{0}(0, *)+\Gamma_{0}(*, *)\right), \\
& n=2 \mu-\Gamma_{2}+\frac{\pi-6}{\pi}\left(-\mu-h+\Gamma_{0}(*, 0)+\Gamma_{0}(*, *)\right) \\
& +\frac{4}{\pi}\left(-\mu-i+\Gamma_{0}(0, *)+\Gamma_{0}(*, *)\right) \\
& =\mu+\frac{2 \mu}{\pi}-\frac{\pi-6}{\pi} h-\frac{4}{\pi} i-\frac{\pi}{6} \Gamma_{\pi / 3}(*, 0)-\frac{\pi-4}{\pi} \Gamma_{2 \pi /(\pi-4)}(0, *) \\
& -\frac{2}{\pi} \Gamma_{\pi}(*, *)-\Gamma_{2}(0,0) \text {. }
\end{aligned}
$$


Hence $n$ is not greater than $\mu+2 \mu / \pi$.

9. Next, let $N$ have at least $r(r>2)$ Sylow divisors each of which is greater than 2: $N=\pi \phi$, where $\pi=p_{1}^{a_{1}} p_{2}^{a_{2}} \cdots p_{r}^{a_{r}}$, the product of powers of $r$ distinct primes, and where $\phi$ is relatively prime to $\pi$. From the $r$ substitutions $(s t)^{N / p_{k}}, k=1,2, \cdots, r$, we have $r$ equations which when added together give

$$
-r \mu-\sum_{k=1}^{r} h_{k}+\sum_{k=1}^{r} k \Gamma_{0}^{(k)}=0
$$

Multiply this equation by $(\pi-2) / r \pi$ and add to

whence

$$
n=2 \mu-\sum_{k=0}^{r} \Gamma_{2}^{(k)}
$$

$$
n=\mu+\frac{2 \mu}{\pi}-\sum_{k=0}^{r} \frac{2 k+\pi(r-k)}{r \pi} \Gamma_{2 r \pi /[2 k+(r-k) \pi]}^{(k)}-\frac{\pi-2}{r \pi} \sum_{k=1}^{r} h_{k} .
$$

If $k=0$,

and if $k=r$,

$$
\frac{2 r \pi}{2 k+(r-k) \pi}=2
$$

$$
\frac{2 r \pi}{2 k+(r-k) \pi}=\pi
$$

since $r>2$, and $\pi-2<r \pi / 3$, we have for $k=1$,

When $1<k<r$,

$$
\frac{2 r \pi}{2+(r-1) \pi}<3 \text {. }
$$

$$
\overline{2 k+(r-k) \pi}<\frac{2 r}{r-k}<2(k+1)<3 \cdot 4 \cdot 5 \cdots \text { to } k \text { factors. }
$$

It follows at once that the greatest possible value of $n$ in this case is $\mu+2 \mu / \pi$. The results of the last three paragraphs may be stated thus:

If the order of st has $r(r>1)$ Sylow divisors, no one of which is the first power of 2 , and whose product we call $\pi$, then the degree of $\{s, t\}$ does not exceed $\mu+2 \mu / \pi$.

10. 'There remains to be investigated the limit of $n$ when $N$ is twice an odd number $(\pi)$ in which there are at least two prime power factors.

If $\pi=p^{\alpha} \cdot 3$, we have already before us

$$
\begin{aligned}
n=\mu+\frac{2 \mu}{\pi}-\frac{4 i}{\pi}-\frac{\pi-6}{\pi} h-\frac{\pi-4}{\pi} \Gamma_{2 \pi /(\pi-4)}(0, *) \\
-\frac{6}{\pi} \Gamma_{\pi / 3}(*, 0)-2 z-\Gamma_{2}(0,0),
\end{aligned}
$$


where $z$ is the number of transitive constituents of degree $2 \pi$ in $\{s, t\}$. Now $(s t)^{\pi}$ gives

$$
2 m+F_{0}=\mu+j,
$$

where $j$ is a positive integer or zero; expanding this,

$$
2 \pi z+F_{0}(*, 0)+F_{0}(0, *)+F_{0}(0,0)=\mu+j-2 m .
$$

Substitute this value of $2 z$ in the expression for $n$ above, and obtain

$$
\begin{aligned}
n=\mu+\frac{\mu}{\pi} & +\frac{2 m}{\pi}-\frac{6}{\pi} E_{\pi / 3}(*, 0)-\frac{\pi-4}{\pi} E_{2 \pi /(\pi-4)}(0, *)-E_{2}(0,0) \\
& -\frac{5}{\pi} F_{2 \pi / 5}(*, 0)-\frac{\pi-5}{\pi} F_{2 \pi /(\pi-5)}(0, *)-\frac{\pi-1}{\pi} F_{2 \pi /(\pi-1)}(0,0) \\
& -\frac{\pi-6}{\pi} h-\frac{4 i}{\pi}-\frac{j}{\pi} .
\end{aligned}
$$

Now

$$
2 m+\Gamma_{0} \leqq \mu+\frac{2 \mu}{\pi},
$$

as has been proved, while $\Gamma_{0} \geqq \mu$. Hence

$$
2 m \leqq \frac{2 \mu}{\pi}, \quad \frac{2 m}{\pi} \leqq \frac{2 \mu}{\pi^{2}},
$$

and therefore

$$
n \leqq \mu+\frac{\mu}{\pi}+\frac{2 \mu}{\pi^{2}}
$$

11. When $N=2 \pi$ is twice an odd number which is the product of two or more prime powers each of which is greater than 3 we may use the formula

$$
n=\mu+\frac{2 \mu}{\pi}-\frac{\pi-2}{r \pi} \sum_{k=1}^{r} h_{k}-\sum_{k=0}^{r-1} \frac{2 k+\pi(r-k)}{r \pi} \Gamma_{2 r \pi /[2 k+(r-k) \pi]}^{(k)}-2 z,
$$

where $z$ is the number of transitive constituents in $\{s, t\}$ of degree $2 \pi$. From $(s t)^{\pi}$ we have

$$
-2 \pi z=-\mu-j+2 m+\sum_{k=0}^{r-1} F_{0}^{(k)}
$$

( $j$ a positive integer or zero), which when substituted for $2 z$ in the preceding expression gives

$$
\begin{aligned}
n=\mu+\frac{\mu}{\pi}+\frac{2 m}{\pi} & -\sum_{k=0}^{r-1} \frac{2 k+\pi(r-k)}{r \pi} E_{2 r \pi /[2 k+(r-k) \pi]}^{(k)} \\
& -\sum_{-0}^{r-1} \frac{2 k-r+\pi(r-k)}{r \pi} F_{2 r \pi /[2 k-r+\pi(r-k)]}^{(k)}-\frac{j}{\pi}-\frac{\pi-2}{r \pi} \sum_{k=1}^{r} h_{k} .
\end{aligned}
$$


It is necessary to show that

$$
\frac{2 r \pi}{2 k-r+\pi(r-k)}<2 \cdot 3 \cdot 5 \cdots \text { to } k+1 \text { factors. }
$$

If $k=0$, the difference $Z-2 \pi /(\pi-1)$ is positive since 6 is the least possible value of $Z$. If $k=1$, we must have $2 r \pi /(2-r+r \pi-\pi)<6$, that is $2 r \pi-3 r-3 \pi+6>0$, or $2(r-2)\left(\pi-\frac{8}{2}\right)+\pi>0$, which is certainly true. Finally for $1<k<r$,

$$
\begin{aligned}
\frac{r \pi}{2 k-r+\pi(r-k)} & <\frac{\pi}{\pi-1} \frac{r}{r-k} \\
& <\left(1+\frac{1}{\pi-1}\right)(k+1) \\
& <2(k+1)<3^{k}<3 \cdot 5 \cdots \text { to } k \text { factors. }
\end{aligned}
$$

Just as in the preceding paragraph we now draw the conclusion that

$$
n \leqq \mu+\frac{\mu}{\pi}+\frac{2 \mu}{\pi^{2}}
$$

When the order of st is twice an odd number $\pi$ divisible by two or more distinct primes the degree of $\{s, t\}$ is not greater than $\mu+\mu / \pi+2 \mu / \pi^{2}$.

12. This result is significant in the proof of the present theorem when $\pi$ is the product of two or more distinct odd primes. In this event it gives a limit for $n$ that is lower than the limit found when the order of $s t$ is $\Pi_{k=1}^{r} p_{k}$, for

In fact,

$$
\frac{1}{\Pi_{k=1}^{r} p_{k}}+\frac{2}{\Pi_{k=1}^{r} p_{k}^{2}}<\frac{1}{\Pi_{k=1}^{r}\left(p_{k}-1\right)} .
$$

$$
\frac{1}{\Pi_{k=1}^{r} p_{k}}+\frac{2}{\Pi_{k=1}^{r} p_{k}^{2}}<\frac{1}{\left(p_{1}-1\right) \Pi_{k=2}^{r} p_{k}} .
$$

It has now been proved that if the order of $s t$ is divisible by the odd primes $p_{1}, p_{2}, \cdots, p_{r}$ its degree is at most $\mu+\mu /\left(p_{1}-1\right)\left(p_{2}-1\right) \cdots\left(p_{r}-1\right)$, and that if its order is $2^{\alpha}, \alpha>1$, its degree does not exceed $\mu+\mu / 2^{a-1}$. Then the theorem follows as stated even when $\{s, t\}$ has regular constituents in letters common to both $s$ and $t$.

The general proposition that the degree of a group of class $\mu$ generated by two non-commutative substitutions of order 2 and degree $\mu$ is at most $\frac{3}{2} \mu$, is covered by this theorem.*

*Bulletin of the American Mathematical Society, vol. 20 (1914), p. 469 . 


\section{MUltiply TRANSITIVE GROUPS}

13. Let $G$ be a $p$ times transitive group, $p$ an odd prime. By hypothesis there is in $G$ a substitution $s$ of order 2 and degree $\mu$ :

$$
s=\left(a_{0} a_{2}\right)\left(a_{3} a_{4}\right) \cdots\left(a_{p-2} a_{p-1}\right) \cdots,
$$

and by reason of the fact that $G$ is more than $p-1$ times transitive, there is in $G$ a second substitution $s^{\prime}$ similar to $s$ :

$$
s^{\prime}=\left(a_{0} a_{3}\right)\left(a_{2} a_{5}\right)\left(a_{4} a_{7}\right) \cdots\left(a_{p-3}-\right) \cdots\left(a_{p-1}\right) .
$$

If the blank space that follows $a_{p-3}$ in $s^{\prime}$ is filled by a letter new to $s$, the product $s s^{\prime}$ has at least one cycle of $p$ letters. Transform $s^{\prime}$ by all the $g_{p-1}$ substitutions of the transitive subgroup of $G$ that leaves fixed the letters $a_{0}, a_{2}, \cdots a_{p-1}$. Each of these $g_{p-1}$ transforms $s^{\prime}, s^{\prime \prime}, \cdots$ has in common with $s$ the $p-2$ letters $a_{0}, a_{2}, \cdots, a_{p-2}$, and $x$ other letters. It is easy to calculate $\sum x$ for these $g_{p-1}$ substitutions $s^{\prime}, s^{\prime \prime}, \cdots$. Any letter, not $a_{0}, a_{2}, \cdots$, or $a_{p-1}$, is found as often in the set as any other. There are in the cycle $\left(a_{p-3}-\right)$ and in the last $\frac{1}{2} \mu-\frac{1}{2}(p-1)$ cycles of the substitutions of the set $s^{\prime}$, $\boldsymbol{s}^{\prime \prime}, \cdots(\mu-p+2) g_{p-1}$ such letters. Hence there are

$$
\frac{(\mu-p+2) g_{p-1}}{n-p+1}
$$

substitutions among $s^{\prime}, s^{\prime \prime}, \cdots$ that contain a given letter (not $a_{0}, a_{2}, \cdots$, $\left.a_{p-1}\right)$. Therefore

(1) $\sum x=(\mu-p+1) \frac{(\mu-p+2) g_{p-1}}{n-p+1}=(\mu-p+1)(\mu-p+2) g_{p}$.

But in the set $s^{\prime}, s^{\prime \prime}, \cdots$ there are exactly $(n-\mu) g_{p}$ substitutions which replace $a_{p-3}$ by a letter new to $s$, and $(\mu-p+1) g_{p}$ that replace $a_{p-3}$ by a letter of $s$. Then

$$
\begin{gathered}
\sum_{(n-\mu) g_{p}}(p-2+x) \geqq \frac{(p-2) \mu}{p-1}(n-\mu) g_{p}, \\
\sum_{(\mu-p+1) g_{p}}(p-2+x) \geqq \frac{1}{2} \mu(\mu-p+1) g_{p} .
\end{gathered}
$$

A word of explanation should perhaps be added. Since all the substitutions $s^{\prime}, s^{\prime \prime}, \cdots$ fix $a_{p-1}$, all are non-commutative with $s$. Moreover, when $s^{\prime}$ (say) replaces $a_{p-3}$ by a letter new to $s, s s^{\prime}$ contains a cycle of degree $p$. It has been proved above that in the latter event $s^{\prime}$ has at most $\mu /(p-1)$ letters new to $s$, whence it follows that the number of letters common to $s^{\prime}$ and $s$ cannot be less than $\mu-\mu /(p-1)=(p-2) \mu /(p-1)$. Then on the addition of (2) and (3),

$$
\sum x \geqq\left(\frac{(p-2) \mu}{p-1}-p+2\right)(n-\mu) g_{p}+\left(\frac{\mu}{2}-p+2\right)(\mu-p+1) g_{p} ;
$$


put for $\sum x$ its value from (1),

$$
\begin{aligned}
(\mu-p+1)(\mu-p+2) g_{p} \geqq \frac{p-2}{p-1}(\mu-p+1)(n-\mu) g_{p} \\
+\left(\frac{\mu}{2}-p+2\right)(\mu-p+1) g_{p},
\end{aligned}
$$

or, on division by $(\mu-p+1) g_{p}$, which is not zero because $\mu>p,{ }^{*}$

whence finally,

$$
\begin{gathered}
\mu-p+2 \geqq \frac{p-2}{p-1}(n-\mu)+\frac{\mu}{2}-p+2, \\
\frac{\mu}{2}+\frac{p-2}{p-1} \mu \geqq \frac{p-2}{p-1} n,
\end{gathered}
$$

$$
\mu \geqq \frac{2 p-4}{3 p-5} n \text {. }
$$

If $p=3$, that is, if $G$ is triply transitive,

$$
\mu \geqq \frac{n}{2} \text {. }
$$

It will be recalled that the holomorph of the elementary abelian group of order $2^{\alpha}(\alpha>1)$ is a triply transitive group of class $2^{\alpha-1}$, in which all the substitutions of degree $2^{a-1}$ are of order 2 .

14. If $p=5$, this limit is

$$
\mu \geqq \frac{3}{5} n \text {. }
$$

Whenever $\mu$ is not divisible by 12 , this may be replaced by

$$
\mu \geqq \frac{3 n+4}{5} \text {. }
$$

For those substitutions $s^{\prime}, s^{\prime \prime}, \cdots$ which replace $a_{p-3}$ by a letter of $s$ cannot have so many as $\mu / 2$ letters in common with $s$ unless such a product as $s^{\prime}$ is of order 6 . The three equations

$$
\begin{aligned}
m+2 y+5 z & =\mu, \\
2 m+6 z & =\mu, \\
3 y+6 z & =\mu,
\end{aligned}
$$

give on solution $z=\mu / 12$. Hence if $\mu$ is not divisible by 12 , no product $s s^{\prime}, s s^{\prime \prime}, \cdots$ is of order 6 , and in consequence

$$
\sum_{(\mu-4) g_{5}}(3+x) \geqq\left(\frac{\mu}{2}+1\right)(\mu-4) g_{5},
$$

* Jordan, Traité des substitutions, 1870, p. 64. 
whence

$$
\mu \geqq \frac{3 n+4}{5}
$$

15. If $p=7$, no product $s s^{\prime}, s s^{\prime \prime}, \cdots$ is of order less than 8 when $s^{\prime}\left(s^{\prime \prime}, \cdots\right)$ replaces $a_{4}$ by a letter of $s$. Then for the $(\mu-6) g_{7}$ substitutions of the set $s^{\prime}, s^{\prime \prime}, \cdots$ which replace $a_{4}$ by a letter of $s$, we have

which added to

$$
\sum_{(\mu-6) g_{7}}(5+x) \geqq \frac{3}{4} \mu(\mu-6) g_{7},
$$

gives on reduction

$$
\sum_{(n-\mu) g_{7}}(5+x) \geqq \frac{5}{6} \mu(n-\mu) g_{7},
$$

$$
\mu \geqq \frac{1}{1} \frac{0}{3} n \text {. }
$$

16. Let it be assumed that $G$ is more than $p$ times transitive, $p$ an odd prime. Then besides

we have

$$
s=\left(a_{0} a_{2}\right)\left(a_{3} a_{4}\right) \cdots\left(a_{p-2} a_{p-1}\right) \cdots\left(a_{p}\right)
$$

$$
\boldsymbol{s}^{\prime}=\left(a_{0} a_{3}\right)\left(a_{2} a_{5}\right) \cdots\left(a_{p-3} a_{p}\right) \cdots\left(a_{p-1}\right) .
$$

The transitive subgroup $G_{p}$ of degree $n-p$ transforms $s^{\prime}$ into a set of $g_{p}$ substitutions $s^{\prime}, \boldsymbol{s}^{\prime \prime}, \cdots$ such that all the products $s s^{\prime}, s s^{\prime \prime}, \cdots$ are of order $p$ or a multiple of $p$. Hence

$$
\sum_{g_{p}}(p-2+x) \geqq \frac{p-2}{p-1} \mu g_{p} .
$$

Any one of the last $\mu-p+1$ letters of $s$ is found in

$$
\frac{(\mu-p+1) g_{p}}{n-p}=(\mu-p+1) g_{p+1}
$$

of the substitutions $s^{\prime}, s^{\prime \prime}, \cdots$. Then

$$
(\mu-p+1)^{2} g_{p+1} \geqq \frac{p-2}{p-1} \mu g_{p}-(p-2) g_{p},
$$

or

$$
(\mu-p+1)^{2} g_{p+1} \geqq \frac{p-2}{p-1}(n-p)(\mu-p+1) g_{p+1},
$$

whence

$$
\mu \geqq \frac{p-2}{p-1} n+\frac{1}{p-1} \text {. }
$$

When applied to quadruply transitive groups this limit is

$$
\mu \geqq \frac{1}{2} n+\frac{1}{2},
$$


and for sextuply transitive groups,

$$
\mu \geqq \frac{3}{4} n+\frac{1}{4} \text {. }
$$

17. The general case may now be very briefly stated. Let the group $G$ be more than $\sigma$ times transitive, where $\sigma$ is the sum of $r(>1)$ distinct odd primes, $p_{1}, p_{2}, \cdots, p_{r}$. When the substitution $s$ is given, $s^{\prime}$, because $G_{\sigma}$ is transitive, may be so written that the first $r$ cycles of $s s^{\prime}$ are of the orders $p_{1}, p_{2}, \cdots, p_{r}$. The substitutions $s$ and $s^{\prime}$ have $\sum_{k=1}^{k=r}\left(p_{k}-2\right)$ common letters in these first $\frac{1}{2} \sum_{k=1}^{k=r}\left(p_{k}-1\right)$ cycles, and $x$ other letters in common. Summing for all the $g_{\sigma}$ substitutions $s^{\prime}, s^{\prime \prime}, \cdots$

$$
\sum x=\left(\mu-\sum_{1}^{r}\left(p_{k}-1\right)\right)^{2} g_{\sigma+1},
$$

and

$$
\sum_{g_{\sigma}}\left(\sum_{1}^{r}\left(p_{k}-2\right)+x\right) \geqq \frac{\prod_{k=1}^{r}\left(p_{k}-1\right)-1}{\prod_{k=1}^{r}\left(p_{k}-1\right)} \mu g_{\sigma}
$$

whence, putting II for $\prod_{1}^{r}\left(p_{k}-1\right)$,

$$
\left(\mu-\sum_{1}^{r}\left(p_{k}-1\right)\right)^{2} \geqq\left[\frac{\Pi-1}{\Pi} \mu-\sum_{1}^{r}\left(p_{k}-2\right)\right]\left(n-\sum_{1}^{r} p_{k}\right),
$$

whence

$$
\mu-\sum_{\mathrm{I}}^{r} p_{k}+r \geqq \frac{\Pi-1}{\Pi}\left(n-\sum_{1}^{r} p_{k}\right)+\frac{r(\Pi+1)-\sum_{1}^{r} p_{k}}{\Pi} \cdot \frac{n-\sum_{1}^{r} p_{k}}{\mu-\sum_{1}^{r}\left(p_{k}-1\right)} .
$$

Since

$$
\begin{gathered}
n-\sum_{1}^{r} p_{k}>\mu-\sum_{1}^{r}\left(p_{k}-1\right), \\
\mu>\frac{\prod_{1}^{r}\left(p_{k}-1\right)-1}{\prod_{1}^{r}\left(p_{k}-1\right)} n+\frac{r}{\prod_{1}^{r}\left(p_{k}-1\right)} .
\end{gathered}
$$

Here the condition $r>1$ must be taken into account because the coefficient of $\left(n-\sum_{k=1}^{k=r} p_{k}\right) /\left(\mu-\sum_{k=1}^{k=r}\left(p_{k}-1\right)\right)$ is zero when $r=1$. Then if a group is more than 8 or more than 15 times transitive, $\mu$ exceeds $\frac{7}{8} n$ or $\frac{47}{4} n$. In connection with this result it might be well to note that a closer study of our group $G$ when 16 times transitive has shown that instead of the limit $\frac{4}{4} \frac{7}{8} n$, the class is actually greater than $\frac{6}{7} \frac{9}{0} n$. Since this appears to be an isolated result depending on the three primes 3,5 , and 7 , I omit the proof. 
18. If $G$ is exactly $\sigma=p_{1}+p_{2}+\cdots+p_{r}$ times transitive, $p_{1}, p_{2}, \cdots, p_{r}$ being distinct odd primes in ascending order of magnitude, $r>1$, we can prove in like manner that

$$
\mu>\frac{\left(\prod_{1}^{r}\left(p_{k}-1\right)-1\right) n+r-1}{\prod_{1}^{r}\left(p_{k}-1\right)+p_{1}-2} .
$$

19. Let $G$ be doubly transitive.* The substitution $s$ of order. 2 and degree $\mu(>3)$ is one of $w$ conjugates. Any transposition occurs exactly

$$
w \mu / n(n-1)
$$

times in this set. The number of possible transpositions in which one letter is displaced by $s$ and the other is fixed by $s$ is $\mu(n-\mu)$, so that if $y$ is the number of substitutions conjugate to $s$ which are not commutative with $s$,

$$
y \geqq \frac{2 w \mu(n-\mu)}{n(n-1)} \text {. }
$$

Since $\mu$ letters may be paired in $\mu(\mu-1) / 2$ ways, the set has $w \mu(\mu-1) / 2$ pairs, and $w \mu(\mu-1) / n(n-1)$ substitutions of the set have a given pair in common. Hence if $x$ is the number of letters any one of the $w$ substitutions has in common with $s$,

$$
\sum_{w} x=w \mu^{2} / n, \quad \sum_{w} x(x-1)=w \mu^{2}(\mu-1)^{2} / n(n-1) .
$$

It is assumed that $\mu$ is less than $n / 2$. Neither $y$ nor $w-y$ is zero. Now

whence

$$
\sum_{w} x^{2}=\sum_{w}\left(x-\frac{1}{w} \sum_{w} x\right)^{2}+\frac{1}{w}\left(\sum_{w} x\right)^{2},
$$

Since

$$
\sum_{w}\left(x-\frac{\mu^{2}}{n}\right)^{2}=\frac{w \mu^{2}(n-\mu)^{2}}{n^{2}(n-1)} \text {. }
$$

and therefore

$$
\begin{gathered}
\sum_{y} x \geqq y \mu / 2, \\
\sum_{y}\left(x-\frac{\mu^{2}}{n}\right)^{2} \geqq y\left(\frac{\mu}{2}-\frac{\mu^{2}}{n}\right)^{2},
\end{gathered}
$$

$$
\sum_{w-y}\left(x-\frac{\mu^{2}}{n}\right)^{2} \leqq \frac{w \mu^{2}(n-\mu)^{2}}{n^{2}(n-1)}-y\left(\frac{\mu}{2}-\frac{\mu^{2}}{n}\right)^{2} .
$$

* This page is a brief sketch of the reasoning given in full in the Bullet in of the American Mathematical Society, 2d series, vol. 20 (1914), pp. $471 \mathrm{ff}$., which see, as well as Bochert, l. c. 
Now

$$
\sum_{w-y}\left(x-\frac{\mu^{2}}{n}\right)^{2} \geqq \frac{1}{w-y}\left[\sum_{w-y}\left(x-\frac{\mu^{2}}{n}\right)\right]^{2} \geqq \frac{1}{w-y} \frac{y^{2} \mu^{2}}{n^{2}}\left(\frac{n}{2}-\mu\right)^{2},
$$

so that

$$
\frac{w \mu^{2}(n-\mu)^{2}}{n^{2}(n-1)}-y\left(\frac{\mu}{2}-\frac{\mu^{2}}{n}\right)^{2} \geqq \frac{1}{w-y} \frac{y^{2} \mu^{2}}{n^{2}}\left(\frac{n}{2}-\mu\right)^{2},
$$

whence finally

$$
\frac{n(n-\mu)}{2 \mu}-\frac{(n-\mu)^{2}}{n-1} \geqq\left(\frac{n}{2}-\mu\right)^{2} .
$$

On solution this inequality gives

\section{STANFORd UNIVERSITY}

$$
\mu>\frac{1}{2} n-\frac{1}{2} \sqrt{n}-1 .
$$

August, 1916 